Research Paper

\title{
Somatic mutations, clinicopathologic characteristics, and survival in patients with untreated breast cancer with bone-only and non-bone sites of first metastasis
}

\author{
Miho Konoㄹ, Takeo Fujii' ${ }^{1}$, Naoko Matsuda ${ }^{1}$, Kenichi Harano1, Huiqin Chen², Chetna Wathoo ${ }^{3}$, Aron Y. \\ Joon ${ }^{2}$, Debu Tripathy ${ }^{1}$, Funda Meric-Bernstam ${ }^{3,4}$, and Naoto T. Ueno ${ }^{1 凶}$ \\ 1. Department of Breast Medical Oncology, The University of Texas MD Anderson Cancer Center, Houston, TX 77030, USA \\ 2. Department of Biostatistics, The University of Texas MD Anderson Cancer Center, Houston, TX 77030, USA \\ 3. Sheikh Khalifa Zayed Al Nahyan Institute for Personalized Cancer Therapy, The University of Texas MD Anderson Cancer Center, Houston, TX 77030, USA \\ 4. Department of Investigational Cancer Therapeutics (Phase I Trials Department), The University of Texas MD Anderson Cancer Center, Houston, TX 77030, \\ USA \\ $\triangle$ Corresponding author: Prof. Naoto T. Ueno, Department of Breast Medical Oncology, Unit 1354, The University of Texas MD Anderson Cancer Center, 1515
} Holcombe Boulevard, Houston, TX 77030, USA. Tel: +1-713-745-6168. Fax: +1-713-794-4385. E-mail: nueno@mdanderson.org

(C) Ivyspring International Publisher. This is an open access article distributed under the terms of the Creative Commons Attribution (CC BY-NC) license (https://creativecommons.org/licenses/by-nc/4.0/). See http://ivyspring.com/terms for full terms and conditions.

Received: 2018.04.22; Accepted: 2018.07.07; Published: 2018.09.08

\begin{abstract}
Background: Bone is the most common site of metastasis of breast cancer. Biological mechanisms of metastasis to bone may be different from mechanisms of metastasis to non-bone sites, and identification of distinct signaling pathways and somatic mutations may provide insights on biology and rational targets for treatment and prevention of bone metastasis. The aims of this study were to compare and contrast somatic mutations, clinicopathologic characteristics, and survival in breast cancer patients with bone-only versus non-bone sites of first metastasis.

Methods: Primary tumor samples were collected before treatment from 389 patients with untreated primary breast cancer and distant metastasis at diagnosis. In each sample, 46 or 50 cancer-related genes were analyzed for mutations by AmpliSeq lon Torrent next-generation sequencing. Fisher's exact test was used to identify somatic mutations associated with bone-only first metastasis. Logistic regression models were used to identify differences in detected somatic mutations, clinicopathologic characteristics, and survival between patients with bone-only first metastasis and patients with first metastasis in non-bone sites only ("other-only first metastasis").

Results: Among the 389 patients, 72 (18.5\%) had bone-only first metastasis, 223 (57.3\%) had other-only first metastasis, and $94(24.2 \%)$ had first metastasis in both bone and non-bone sites. The most commonly mutated genes were TP53 $(\mathrm{N}=103)$, PIK3CA $(\mathrm{N}=79)$, AKT $(\mathrm{N}=13)$, and PTEN $(\mathrm{N}=2)$. Compared to patients with other-only first metastasis, patients with bone-only first metastasis had higher rates of hormone-receptor-positive disease, non-triple-negative subtype, and lower grade (grade 1 or 2; Nottingham grading system) (all three comparisons, $p<0.001$ ); had a lower ratio of cases of invasive ductal carcinoma to cases of invasive lobular carcinoma $(p=0.002)$; and tended to have a higher 5 -year overall survival (OS) rate (78.2\% [ $95 \%$ confidence interval $(\mathrm{Cl}), 68.6 \%-89.0 \%$ ] vs $55.0 \%$ [ $95 \% \mathrm{Cl}, 48.1 \%-62.9 \%]$; $p=0.051)$. However, in the subgroup of patients with TP53 mutation and in the subgroup of patients with PIK3CA mutation, OS did not differ between patients with bone-only and other-only first metastasis $(p=0.49$ and $p=0.68$, respectively). In univariate analysis, the rate of TP53 mutation tended to be lower in patients with bone-only first metastasis than in those with other-only first metastasis $(15.3 \%$ vs $29.1 \%$; $p=0.051)$. In multivariate analysis, TP53 mutation was not significantly associated with site of first metastasis $(p=0.54)$ but was significantly associated with hormone-receptor-negative disease $(p<0.001)$.

Conclusions: We did not find associations between somatic mutations and bone-only first metastasis in patients with untreated breast cancer. Patients with bone-only first metastasis tend to have longer OS than patients with other-only first metastasis. More comprehensive molecular analysis may be needed to further understand the factors associated with bone-only metastatic disease in breast cancer.
\end{abstract}

Key words: breast cancer, bone metastasis, somatic mutation, prognostic factor, TP53, PIK3CA 


\section{Introduction}

Bone is the most common site of breast cancer metastasis, and bone metastases are present in $65 \%$ to $75 \%$ of patients with metastatic breast cancer [1-4]. Moreover, bone has been reported to be the most common site of first distant relapse of breast cancer [5, 6]. Bone metastases of breast cancer are generally incurable, but survival times are longer when sites of metastasis are limited to bone than when breast cancer has metastasized to other sites. Bone metastasis of breast cancer can cause skeletal-related events such as bone pain, hypercalcemia, pathologic fracture, and compression of the spinal cord, all of which decrease quality of life [7], and skeletal-related events are strongly associated with mortality among breast cancer patients [8].

Clinical management of bone metastasis from breast cancer is challenging. Palliative radiation therapy has been used for relief of bone pain and prevention of skeletal-related events in patients with bone metastasis [9]. Bone-targeted therapies such as zoledronic acid and denosumab, a monoclonal antibody against receptor activator of nuclear factor kappa-B ligand (RANK-L), have been the standard drugs used to manage bone metastasis and reduce the frequency of skeletal-related events associated with poor quality of life [10]. Recently, radium-223 dichloride alpha-particle (radium-223) therapy, which was approved for symptomatic bone metastasis from castration-resistant prostate cancer [11], increased survival in a breast cancer mouse model of bone metastasis [12]. This promising treatment was tolerated in patients with bone disease who experienced disease progression during endocrine therapy in a phase IIa study [13]. Phase II studies of radium-223 in breast cancer patients with bone metastasis are ongoing (NCT02258464, NCT02258451, NCT02366130). However, no drugs have been identified that target bone metastasis and extend survival in breast cancer patients with bone metastasis.

Identification of somatic mutations and clinicopathological characteristics that are more prevalent in patients with bone-only first metastasis than in patients with first metastasis in non-bone sites only ("other-only first metastasis") could identify novel targets for treatment and prevention of bone metastasis from breast cancer. However, whether specific mutations are associated with breast cancer metastasis to bone only as the first site of metastasis is poorly understood. Thus, we compared and contrasted somatic hotspot mutations, clinicopathologic characteristics, and survival in patients with untreated primary breast cancer with bone-only versus other-only first metastasis.

\section{Methods}

\section{Patient Selection}

Patients included in the study were drawn from a larger group of patients whose tumor samples were examined for gene mutations at The University of Texas MD Anderson Cancer Center during the period from March 2012 through December 2014 as part of Institutional Review Board-approved protocol PA11-0852. All patients provided informed consent before enrollment in PA11-0852. Inclusion criteria for PA11-0852 were a diagnosis of cancer, an available tumor sample previously collected from either a primary tumor or a metastasis, and potential to benefit from somatic genomic testing. To be eligible for the study reported herein, patients had to have breast cancer with distant metastasis identified at the time of diagnosis of the primary tumor. They also had to have a primary breast tumor sample collected before treatment to avoid any influence of treatment on mutations; treatment has previously been reported to induce TP53 and PIK3CA mutations [14]. Finally, patients had to have information available about the first site of metastasis.

We identified 922 patients with distant metastasis at diagnosis of breast cancer who had samples obtained before treatment and submitted for molecular testing in PA11-0852 during the study period. Of these 922 patients, 482 patients had specimens obtained from a metastasis and were excluded; 440 had specimens obtained from the primary tumor. From these 440 patients, we excluded the 51 patients for whom no information was available about the site of first metastasis, leaving 389 patients in this study.

\section{Tumor Sample Analysis}

Specimens included formalin-fixed, paraffinembedded core needle biopsy specimens and tumor resection specimens. Tumor-rich areas were manually macrodissected, and only cases with more than $20 \%$ tumor cellularity were included. Tumors were classified as grade 1 (low grade), grade 2 (intermediate grade), or grade 3 (high grade) according to the Nottingham grading system, which is based on a combination of nuclear grade, tubule formation, and mitotic rate. Tumors were defined as hormone receptor positive if immunohistochemical (IHC) staining of archival tumor tissue samples for estrogen receptor (antibody clone 6F11-Novacastra) or progesterone receptor (antibody clone PgR1294-DAKO) showed expression in at least $10 \%$ of cells [15]. HER2-positive status was defined as 3+ IHC staining or a HER2/CEP17 ratio $\geq 2.0$ on fluorescence in situ hybridization [16]. All histologic diagnoses and grading and IHC staining results were reviewed by 
the breast pathology service at MD Anderson Cancer Center.

\section{Genomic Analysis}

In each sample, 46 or 50 commonly mutated cancer-related genes were selectively analyzed for mutations by AmpliSeq Ion Torrent next-generation sequencing as previously described $[17,18]$. This is a multiplex PCR-based library preparation method by which 190 regions (70-150 bp) that encompass 740 mutational hotspots in the coding sequence of 46 or 50 cancer-related genes are analyzed for mutations using multiple markers of detection [17]. The following genes were included in the 46-gene panel: $A B L 1$, $A K T 1, A L K, A P C, A T M, B R A F, C D H 1, C D K N 2 A$, CSF1R, CTNNB1, EGFR, ERBB2, ERBB4, FBXW7, FGFR1, FGFR2, FGFR3, FLT3, GNAS, HNF1A, HRAS, IDH1, JAK2, JAK3, KDR, KIT, KRAS, MET, MLH1, MPL, NOTCH1, NPM1, NRAS, PDGFRA, PIK3CA, PTEN, PTPN11, RB1, RET, SMAD4, SMARCB1, SMO, SRC, STK11, TP53, and VHL [19]. The 50-gene panel included those 46 genes plus EZH2, GNA11, GNAQ, and IDH 2 .

\section{Statistical Analysis}

Overall survival (OS) was measured from the date of diagnosis of primary breast cancer to the date of death or last follow-up. OS was calculated by using Kaplan-Meier estimators, and the log-rank test was used to compare the Kaplan-Meier curves. Continuous variables were evaluated by using the Cox proportional hazards model. Rates of somatic mutations were compared between patient groups by using Fisher's exact test. The associations between bone metastasis and clinicopathological variables of interest were evaluated; these variables included age at diagnosis, race, sex, menopausal status at diagnosis, histology, grade, and subtype (hormone receptor positive or negative, HER2 positive or negative, and triple-negative or not). The association between mutation status and clinicopathologic variables of interest was estimated by using logistic regression models. Variables statistically significant $(p$ $<0.05$ ) in univariate analysis and site of first metastasis were selected for multivariate analysis.

\section{Results}

\section{Patient Characteristics}

Patient demographic and clinical characteristics by site of first metastasis are shown in Table 1. Among the 389 patients in the study, $72(18.5 \%)$ had bone-only first metastasis, $223(57.3 \%)$ had other-only first metastasis, and 94 (24.2\%) had first metastasis in both bone and non-bone sites. Compared to patients with other-only first metastasis, patients with bone-only first metastasis had higher rates of lower grade (grade 1 and 2) $(p<0.001)$, hormone-receptor-positive disease $(p<0.001)$, and non-triple-negative subtype $(p<0.001)$ and a lower ratio of cases of invasive ductal carcinoma to cases of invasive lobular carcinoma $(p=0.002)$. Rates of HER2 positivity did not differ between patients with bone-only and other-only first metastasis $(p=0.10)$.

\section{Overall Survival}

OS for the entire study group and by site of metastasis and mutation status is shown in Table 2. The median OS in patients with bone-only first metastasis was 126 months, and the median OS in patients with other-only first metastasis was 85 months. The 5-year OS rate tended to be higher for patients with bone-only first metastasis than for patients with other-only first metastasis $(78.2 \%$ vs $55.0 \% ; p=0.051)$. However, in the subgroup of patients with TP53 mutation and in the subgroup of patients with PIK3CA mutation, OS did not differ between patients with bone-only and other-only first metastasis $(p=0.49$ and $p=0.68$; respectively). The numbers of patients with AKT1 and PTEN mutations were too small to permit subgroup analysis of differences in OS between patients with bone-only and other-only first metastasis among patients with these mutations.

\section{Mutation Analysis}

Somatic mutations in four genes, TP53, PIK3CA, $A K T$, and PTEN, were detected among the 389 patients. Mutation rates for the overall study group and subgroups defined by site of first metastasis are shown in Table 3. In the overall study group (389 patients), TP53 mutations were the most common, detected in 103 patients (26.5\%); PIK3CA mutations were detected in 79 patients $(20.3 \%)$, AKT mutations were detected in 13 patients $(3.3 \%)$, and PTEN mutations were detected in two patients $(0.5 \%)$. In the 72 patients with bone-only first metastasis, PIK3CA mutations were the most common, detected in 18 patients (25.0\%); TP53 mutations were detected in 11 patients $(15.3 \%)$ and $A K T$ mutations were detected in two patients $(2.8 \%)$. In univariate analysis, the rate of TP53 mutation tended to be lower in patients with bone-only first metastasis than in those with other-only first metastasis $(15.3 \%$ vs $29.1 \% ; p=0.051)$, but the rates of PIK3CA, AKT, and PTEN mutations were not significantly different between patients with bone-only and other-only first metastasis. In multivariate logistic regression analysis, TP53 mutation was not significantly associated with site of first metastasis $(p=0.54)$ but was significantly associated with hormone-receptor-negative disease $(p<0.001)$ (Table 4). 
Table 1: Demographic and clinical characteristics by site of first metastasis in 389 patients with untreated primary breast cancer with distant metastasis.a

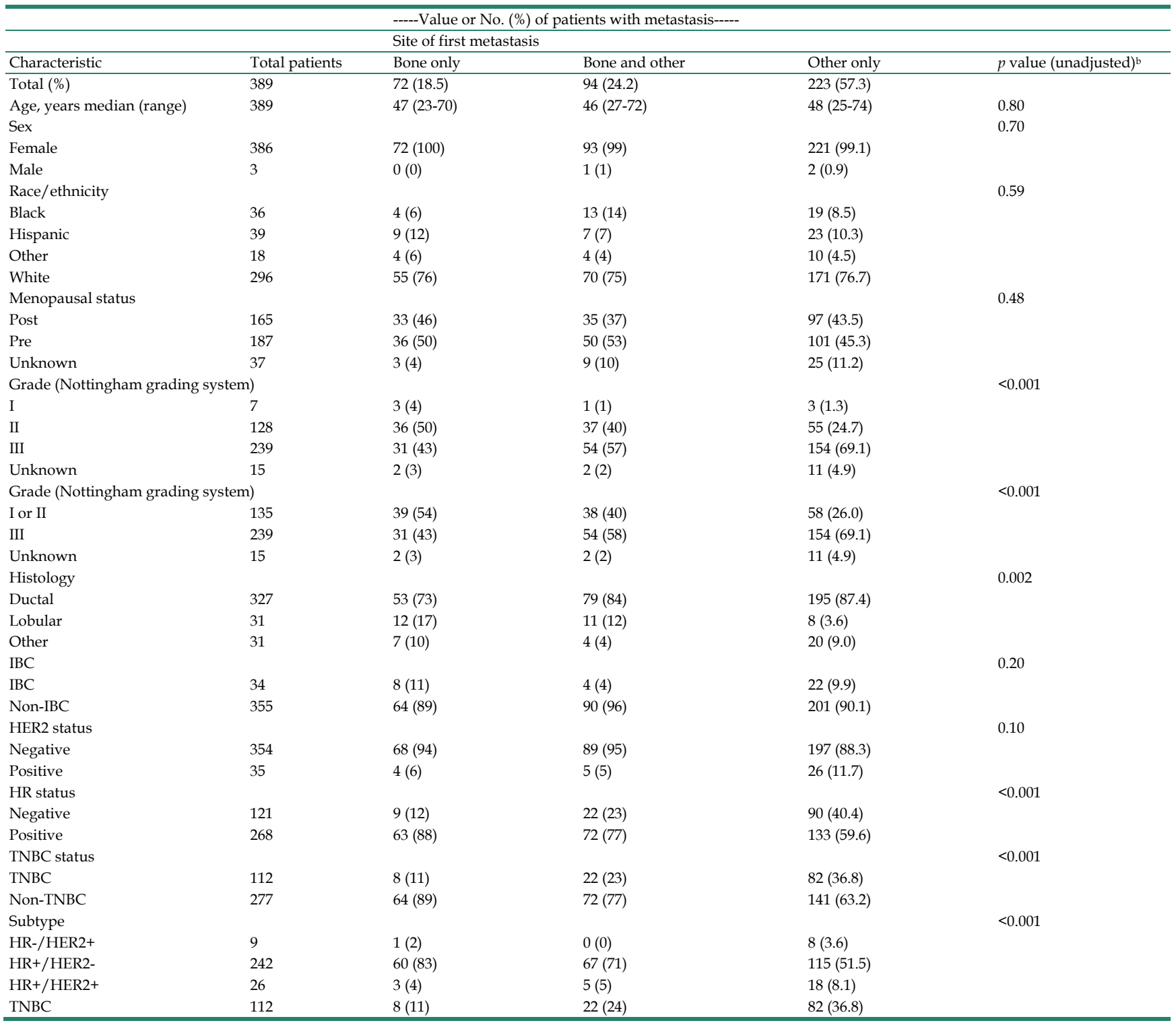

Abbreviations: HR, hormone receptor; IBC, inflammatory breast cancer; TNBC, triple-negative breast cancer.

aValues in table are number of patients (percentage) unless otherwise indicated.

bFor comparison between patients with bone-only and other-only metastases.

Table 2: Overall survival (OS) by site of first metastasis and mutation status.

\begin{tabular}{lll}
\hline Site of first metastasis & Median OS $(95 \% \mathrm{CI})$, months & 5-year OS rate $(95 \% \mathrm{CI}), \%$ \\
\hline $\begin{array}{ll}\text { All patients, regardless of mutation status } \\
\text { All }\end{array}$ & $96(76-119)$ & $62.2(57.3-67.6)$ \\
Bone only & $126(87-\mathrm{NR})$ & $78.2(68.6-89.0)$ \\
Bone and other & $73(50-117)$ & $58.0(48.1-69.9)$ \\
Other only & $85(59-119)$ & $55.0(48.1-62.9)$ \\
Patients with TP53 mutation & & \\
Bone only & $47(32-\mathrm{NR})$ & $48.5(24.8-94.9)$ \\
Bone and other & $67(37-\mathrm{NR})$ & $52.4(34.4-79.9)$ \\
Other only & $59(46-130)$ & $47.3(33.4-67.0)$ \\
Patients with PIK3CA mutation & & \\
Bone only & $98(72-\mathrm{NR})$ & $86.7(71.1-100)$ \\
Bone and other & $96(73-\mathrm{NR})$ & $80.0(62.1-100)$ \\
Other only & $88(53-\mathrm{NR})$ & $57.9(43.6-76.9)$
\end{tabular}

Abbreviations: $\mathrm{CI}$, confidence interval; NR, not reached.

aFor comparison between patients with bone-only and other-only metastases. 
Table 3: Mutations by site of first metastasis.

\begin{tabular}{llllll}
\hline \multicolumn{5}{c}{ No. (\%) of patients with metastasis } \\
\hline $\begin{array}{l}\text { Patient } \\
\text { subgroup }\end{array}$ & $\begin{array}{l}\text { Total no. } \\
\text { of patients }\end{array}$ & Bone only & $\begin{array}{l}\text { Bone and } \\
\text { other }\end{array}$ & Other only & $\begin{array}{l}p \text { value } \\
\text { (unadjusted) }\end{array}$ \\
\hline $\begin{array}{l}\text { All patients } \\
\text { TP53 }\end{array}$ & 789 & 72 & 94 & 223 & 0.051 \\
Wild-type & 286 & $61(85)$ & $67(71)$ & $158(70.9)$ & \\
Mutated & 103 & $11(15)$ & $27(29)$ & $65(29.1)$ & 0.559 \\
PIK3CA & & & & & \\
Wild-type & 310 & $54(75)$ & $77(82)$ & $179(80.3)$ & \\
Mutated & 79 & $18(25)$ & $17(18)$ & $44(19.7)$ & 0.176 \\
$\begin{array}{l}\text { AKT1 } \\
\text { Wild-type }\end{array}$ & 376 & $70(97)$ & $88(94)$ & $218(97.8)$ & \\
Mutated & 13 & $2(3)$ & $6(6)$ & $5(2.2)$ & \\
PTEN & & & & & \\
Wild-type & 387 & $72(100)$ & $93(99)$ & $222(99.6)$ & \\
Mutated & 2 & $0(0)$ & $1(1)$ & $1(0.4)$ & \\
\hline
\end{tabular}

Table 4: Logistic regression analysis of relationship between TP53 mutation and site of first metastasis and other clinicopathologic characteristics.

\begin{tabular}{lll}
\hline Parameter & OR $(95 \% \mathrm{CI})$ & $p$ value (adjusted) \\
\hline $\begin{array}{l}\text { Site of first metastasis (other only vs } \\
\text { bone only) }\end{array}$ & $\mathbf{1 . 2 7}(\mathbf{0 . 6 1 - 2 . 8 0})$ & $\mathbf{0 . 5 4 4}$ \\
Grade (III vs I or II) & $2.01(1.12-3.72)$ & 0.022 \\
HER2 status (positive vs negative) & $0.71(0.27-1.66)$ & 0.448 \\
HR status (positive vs negative) & $0.35(0.21-0.59)$ & $<0.001$ \\
Histology (lobular vs ductal) & $0.35(0.05-1.30)$ & 0.174 \\
Histology (other vs ductal) & $0.60(0.20-1.53)$ & 0.308
\end{tabular}

Abbreviations: CI; confidence interval; $\mathrm{HR}$, hormone receptor; $\mathrm{OR}$; odds ratio.

\section{Discussion}

Mutational profiles have revealed mutations whose frequency differs between primary and metastatic breast cancer. These mutations might be targeted therapeutically and might be associated with resistance to existing therapies in metastatic breast cancer. For example, PIK3CA mutation status, which is related to the PI3K/Akt/mTOR signaling pathway, showed discordance between primary and metastatic breast cancer [20], and ESR1 and RB1 mutations were detected in metastatic breast cancer but infrequently mutated in primary breast cancer [21]. Furthermore, several studies have demonstrated that signaling pathways and somatic mutations are associated with bone metastasis in breast cancer, and these pathways and mutations may be rational targets for treatment and prevention of bone metastasis. For example, a gene-expression signature of c-Src activation is associated with bone metastasis in breast cancer, and activation of the Src signaling pathway increases the predilection of a subset of primary breast cancer cells to metastasize to bone [22]. Runt-related transcription factor 2 (RUNX2) significantly contributes to bone metastasis in breast cancer [23-25]. SNAI2 is activated by ERa signaling and promoted bone metastasis [26], while SNAI2 is negatively correlated with ERa signaling and suppressed non-bone metastasis in breast cancer [22, 26, 27].
In our current study, we did not find associations between somatic mutations and bone-only first metastasis in patients with untreated breast cancer. We did confirm previous analyses showing that compared to first metastasis to non-bone site, first metastasis to bone was associated with well-differentiated tumors, hormone-receptorpositive disease, and a lower ratio of cases of invasive ductal carcinoma to cases of invasive lobular carcinoma, all of which are known favorable tumor factors [6, 28-32]. Although OS of patients with bone-only first metastasis tended to be longer than that of patients with other-only first metastasis, OS did not differ between patients with bone-only and other-only first metastasis in the subgroup of patients with TP53 mutation or in the subgroup of patients with PIK3CA mutation.

Among the 72 patients with bone-only first metastasis, we detected mutations in three genes, TP53, PIK3CA, and AKT. Our findings with respect to TP53 and PIK3CA mutations agree with previous studies, which have shown that these mutations are the most common mutations not only in primary breast cancer [33] but also in metastatic breast cancer $[18,21]$. Although some patients in the previous studies showing TP53 and PIK3CA mutations in metastatic breast cancer were exposed to treatment before tumor specimens were obtained for mutational analysis $[18,21]$, the results were the same as in our patients with metastases and untreated primary breast cancer. In our study, the rate of TP53 mutation tended to be lower in patients with bone-only first metastasis than in those with other-only first metastasis in univariate analysis. However, in multivariate analysis, TP53 mutation was not significantly associated with site of first metastasis but was associated with hormone-receptor-negative disease. Our results appear to be in agreement with previous reports that TP53 mutation was associated with breast cancer subtype, especially hormone-receptor-negative disease [18, 34].

Our study has several limitations. First, we evaluated 46 or 50 genes reported as frequently mutated on the basis of next-generation sequencing. We may have missed some as yet unidentified somatic mutations related to bone-only metastasis. RUNX2 and SNAI2, which may significantly contribute to bone metastasis in breast cancer [23-26], were not evaluated in our current study. Second, the restaging or reimaging strategy was not uniform across patients in the study. We may have missed some cases of bone-only metastasis or other-only metastasis. Third, we could not detect copy number variations or amplifications. Fourth, we were not able to perform subgroup analyses in patients with AKT1 
and PTEN mutations because of the small number of patients with these mutations. Finally, this was a retrospective study, and selection bias was unavoidable given the population of patients who were enrolled in the original genomic study. As far as we know, our study is the first study to analyze for somatic mutations associated with bone metastasis in patients with untreated primary breast cancer. Our results may influence future molecular analyses of bone metastasis from breast cancer.

In conclusion, we did not find unique somatic mutations associated with bone-only first metastasis of breast cancer or OS in patients with bone-only first metastasis of breast cancer. The long OS in some patients with breast cancer with bone-only first metastasis may indicate that strategies to target bone metastasis and skeletal-related events would help improve quality of life for these patients. More comprehensive molecular analysis may be needed to further understand the factors associated with bone-only metastatic disease in breast cancer.

\section{Acknowledgments}

The authors appreciate the assistance of Stephanie P. Deming of the Department of Scientific Publications at MD Anderson Cancer Center.

\section{Funding}

This work was supported by the Morgan Welch Inflammatory Breast Cancer Research Program, a grant from the State of Texas Rare and Aggressive Breast Cancer Research Program, the Cancer Prevention and Research Institute of Texas (RP1100584), the Sheikh Khalifa Bin Zayed Al Nahyan Institute for Personalized Cancer Therapy, National Center for Advancing Translational Sciences grant UL1 TR000371 (supporting the Center for Clinical and Translational Sciences), National Institutes of Health grant P30CA016672, and Japan Cancer Society.

\section{Competing Interests}

The authors have declared that no competing interest exists.

\section{References}

1. Coleman RE. Metastatic bone disease: clinical features,pathophysiology and treatment strategies. CANCER TREATMENT REVIEWS. 2001; 27: 165-176.

2. Mackiewicz-Wysocka M, Pankiwska M, Wysocki PJ. Progress in the treatment of bone metastases in cancer patients. Expert Opin Investig Drugs. 2012; 21(6):785-95.

3. Coleman RE, Smith P, Rubens RD. Clinical course and prognostic factors following bone recurrence from breast cancer. Br J Cancer. 1998; 77: 336-340.

4. Savci-Heijink CD, Halfwerk H, Hooijer GK, et al. Retrospective analysis of metastatic behaviour of breast cancer subtypes. Breast Cancer Res Treat. 2015; 150: 547-557.

5. McNeil BJ. Value of bone scanning in neoplastic disease. Semin Nucl Med. 1984; 14: 277-286.
6. Coleman RE, Rubens RD. The clinical course of bone metastases from breast cancer. Br J Cancer. 1987; 55: 61-66.

7. Weinfurt KP, Castel LD, Li Y, et al. Health-related quality of life among patients with breast cancer receiving zoledronic acid or pamidronate disodium for metastatic bone lesions. Med Care. 2004; 42: 164-175.

8. Sathiakumar N, Delzell E, Morrisey MA, et al. Mortality following bone metastasis and skeletal-related events among women with breast cancer: a population-based analysis of U.S. Medicare beneficiaries, 1999-2006. Breast Cancer Res Treat. 2012; 131: 231-238.

9. Sze WM, Shelley M, Held I, et al. Palliation of metastatic bone pain: single fraction versus multifraction radiotherapy - a systematic review of the randomised trials. Cochrane Database Syst Rev. 2004; (2): Cd004721.

10. Eckhardt BL, Francis PA, Parker BS, et al. Strategies for the discovery and development of therapies for metastatic breast cancer. Nat Rev Drug Discov. 2012; 11: 479-497.

11. Hoskin P, Sartor O, O'Sullivan JM, et al. Efficacy and safety of radium-223 dichloride in patients with castration-resistant prostate cancer and symptomatic bone metastases, with or without previous docetaxel use: a prespecified subgroup analysis from the randomised, double-blind, phase 3 ALSYMPCA trial. Lancet Oncol. 2014; 15: 1397-1406.

12. Suominen MI, Rissanen JP, Kakonen R, et al. Survival benefit with radium-223 dichloride in a mouse model of breast cancer bone metastasis. J Natl Cancer Inst. 2013; 105: 908-916.

13. Coleman R, Aksnes AK, Naume B, et al. A phase IIa, nonrandomized study of radium-223 dichloride in advanced breast cancer patients with bone-dominant disease. Breast Cancer Res Treat. 2014; 145: 411-418.

14. Jiang YZ, Yu KD, Bao J,et al. Favorable prognostic impact in loss of TP53 and PIK3CA mutations after neoadjuvant chemotherapy in breast cancer. Cancer Res. 2014; 74(13): 3399-407.

15. Hammond ME, Hayes DF, Dowsett M, et al. American Society of Clinical Oncology/College of American Pathologists guideline recommendations for immunohistochemical testing of estrogen and progesterone receptors in breast cancer. Arch Pathol Lab Med. 2010; 134(6): 907-22.

16. Carlson RW, Moench SJ, Hammond ME, et al. HER2 testing in breast cancer: NCCN Task Force report and recommendations. J Natl Compr Canc Netw. 2006 Jul; 4 Suppl 3: S1-22; quiz S23-24.

17. Singh RR, Patel K, Routbort MJ, et al. Clinical validation of a next-generation sequencing screen for mutational hotspots in 46 cancer-related genes. J Mol Diagn. 2013; Sep;15(5): 607-22.

18. Basho RK, de Melo Gagliato D, Ueno NT, et al. Clinical outcomes based on multigene profiling in metastatic breast cancer patients. Oncotarget. 2016; 7: 76362-76373.

19. Kanagal-Shamanna R, Portier BP, Singh RR, et al. Next-generation sequencing-based multi-gene mutation profiling of solid tumors using fine needle aspiration samples: promises and challenges for routine clinical diagnostics. Mod Pathol. 2014; Feb;27(2): 314-27.

20. Gonzalez-Angulo AM, Ferrer-Lozano J, Stemke-Hale K, et al. PI3K pathway mutations and PTEN levels in primary and metastatic breast cancer. Mol Cancer Ther. 2011; 10: 1093-1101.

21. Lefebvre $\mathrm{C}$, Bachelot $\mathrm{T}$, Filleron $\mathrm{T}$, et al. Mutational Profile of Metastatic Breast Cancers: A Retrospective Analysis. PLoS Med. 2016 Dec 27; 13(12): e1002201.

22. Zhang $\mathrm{XH}$, Wang $\mathrm{Q}$, Gerald $\mathrm{W}$, et al. Latent bone metastasis in breast cancer tied to Src-dependent survival signals. Cancer Cell. 2009; 16: $67-78$.

23. Onodera Y, Miki Y, Suzuki T, et al. Runx2 in human breast carcinoma: its potential roles in cancer progression. Cancer Sci. 2010; 101: 2670-2675.

24. Shore P. A role for Runx 2 in normal mammary gland and breast cancer bone metastasis. J Cell Biochem. 2005; 96: 484-489.

25. Pratap J, Wixted JJ, Gaur T, et al. Runx2 transcriptional activation of Indian Hedgehog and a downstream bone metastatic pathway in breast cancer cells. Cancer Res. 2008; 68: 7795-7802.

26. Chimge NO, Baniwal SK, Little GH, et al. Regulation of breast cancer metastasis by Runx 2 and estrogen signaling: the role of SNAI2. Breast Cancer Res. 2011; 13(6): R127.

27. Hess KR, Pusztai L, Buzdar AU, et al. Estrogen receptors and distinct patterns of breast cancer relapse. Breast Cancer Res Treat. 2003; 78: 105-118.

28. Hayashi N, Iwamoto T, Qi Y, et al. Bone metastasis-related signaling pathways in breast cancers stratified by estrogen receptor status. J Cancer. 2017; 8: 1045-1052.

29. James JJ, Evans AJ, Pinder SE, et al. Bone metastases from breast carcinoma: histopathological - radiological correlations and prognostic features. Br J Cancer. 2003; 89: 660-665.

30. Smid M, Wang Y, Klijn JG, et al. Genes associated with breast cancer metastatic to bone. J Clin Oncol. 2006; 24: 2261-2267. 
31. Elston CW, Blamey RW, Johnson J, et al. The relationship of oestradiol receptor (ER) and histological tumour differentiation with prognosis in human primary breast carcinoma. Eur J Cancer. 1980; Suppl 1: 59-62.

32. Wasif N, Maggard MA, Ko CY, et al. Invasive lobular vs. ductal breast cancer: a stage-matched comparison of outcomes. Ann Surg Oncol. 2010; 17: $1862-1869$.

33. Cancer Genome Atlas Network. Comprehensive molecular portraits of human breast tumours. Nature. 2012; Oct 4; 490 (7418): 61-70.

34. Shah SP, Roth A, Goya R, et al. The clonal and mutational evolution spectrum of primary triple-negative breast cancers. Nature. 2012; 486: 395-399. 